\title{
A ENFERMAGEM FORENSE E SEUS CONTEÚDOS CURRICULARES NOS CURSOS DE GRADUAÇÃO EM ENFERMAGEM
}

\section{FORENSIC NURSING AND ITS CURRICULUM CONTENT IN UNDERGRADUATE NURSING COURSES \\ ENFERMERÍA FORENSE Y SU CONTENIDO CURRICULAR EN CURSOS DE PREGRADO EN ENFERMERÍA}

Jhuliano Silva Ramos de Souza ${ }^{1}$, Christianne Alves Pereira Calheiros ${ }^{2}$, Fábio de Souza Terra ${ }^{3}$, Andreia Cristina Barbosa Costa ${ }^{4}$, Sueli de Carvalho Vilela².

\section{RESUMO}

Objetivo: Identificar os conteúdos curriculares de enfermagem forense, nos cursos de graduação em Enfermagem. Método: Tratase de uma pesquisa quantitativa, descritiva e exploratória, realizada com coordenadores dos cursos de Enfermagem de 88 Instituições de Ensino Superior. Utilizou-se o Teste Exato de Fisher, para verificar a significância entre as variáveis dependentes (Instituições de Ensino Superior públicas e privadas). Resultados: Ao cruzar as variáveis, as Instituições de Ensino Superior privadas (76,5\%) tiveram mais respostas em relação às públicas $(50,0 \%)$ que fornecem tais conteúdos nas suas receptivas disciplinas. Para tal, os conteúdos de enfermagem forense por Instituições de Ensino Superior foram sobre pessoas que sofreram violência no trabalho, vítimas de desastre em massa, pessoas sob uso e abuso de álcool e outras drogas e internações compulsórias. Conclusão: Foi possível identificar os conteúdos ministrados nos cursos de Enfermagem, salientando-se a necessidade da existência de uma disciplina específica de enfermagem forense a ser ofertada no projeto pedagógico de curso.

Descritores: Enfermagem Forense; Currículo; Educação; Educação Superior.

\section{ABSTRACT}

Objective: To identify the curricular content of forensic nursing in undergraduate nursing courses. Method: This is a quantitative, descriptive and exploratory research carried out with coordinators of the Nursing courses of 88 Higher Education Institutions. Fisher's exact test was used to verify the significance between the dependent variables (public and private higher education institutions). Results: When crossing the variables, private Higher Education Institutions (76.5\%) had more responses than public ones (50.0\%) that provide such content in their receptive disciplines. To this end, the content of forensic nursing by Higher Education Institutions was about people who suffered violence at work, victims of mass disaster, people under the use and abuse of alcohol and other drugs and compulsory hospitalizations. Conclusion: It was possible to identify the contents taught in nursing courses, emphasizing the need for the existence of a specific forensic nursing discipline to be offered in the course pedagogical project.

Descriptors: Forensic Nursing; Curriculum; Education; Higher Education.

\section{RESUMEN}

Objetivo: Identificar el contenido curricular de la enfermería forense en los cursos de pregrado en enfermería. Método: Se trata de una investigación cuantitativa, descriptiva y exploratoria, realizada con coordinadores de los cursos de Enfermería de 88 Instituciones de Educación Superior. La prueba exacta de Fisher se utilizó para verificar la importancia entre las variables dependientes (instituciones de educación superior públicas y privadas). Resultados: Al cruzar las variables, las instituciones privadas de educación superior (76.5\%) tuvieron más respuestas que las públicas (50.0\%) que proporcionan dicho contenido en sus disciplinas receptivas. Con este fin, el contenido de enfermería forense por parte de las instituciones de educación superior fue sobre personas que sufrieron violencia en el trabajo, víctimas de desastres masivos, personas bajo el uso y abuso de alcohol y otras drogas y hospitalizaciones obligatorias. Conclusión: Fue posible identificar los contenidos enseñados en los cursos de Enfermería, enfatizando la necesidad de ofrecer una disciplina específica de enfermería forense en el proyecto del curso pedagógico.

Descriptores: Enfermería Forense; Curriculum; Educación; Educación Superior.

${ }^{1}$ Enfermeiro. Mestre em Enfermagem pela Universidade Federal de Alfenas. ${ }^{2}$ Doutora em Ciências. Docente na Universidade Federal de Alfenas. ${ }^{3}$ Doutor em Ciências. Docente na Universidade Federal de Alfenas. ${ }^{4}$ Pós-doutorado em Enfermagem. Docente na Universidade Federal de Alfenas.

\section{Como citar este artigo:}

Souza JSR, Calheiros CAP, Terra FS, et al. A Enfermagem Forence e seus conteúdos curriculares nos cursos de graduação em enfermagem. Revista de Enfermagem do centro Oeste Mineiro. 2020;10:e3635. [Access ; Available in DOI: http://doi.org/10.19175/recom.v10i0.3635 


\section{INTRODUÇÃO}

Nas últimas décadas, o ensino de enfermagem teve modificações no âmbito epidemiológico, econômico, social e cultural, o qual foi substituído por um modelo científico singular e dinâmico, com o intuito de formar enfermeiros não só generalistas, mas também pesquisadores, educadores qualificados em atuar nos seus diferentes cenários da profissão ${ }^{(1)}$,por meio de programas de pós-graduação voltados para a qualificação profissional, inseridos no desenvolvimento de pesquisas que impactam positivamente na sociedade ${ }^{(2)}$.

No entanto, a formação do enfermeiro com titulação bacharelado é regida pelas Diretrizes Curriculares Nacionais dos Cursos de Enfermagem(DCN/ENF) ${ }^{(3)}$, o qual traz o perfil generalista, humanista, crítico e reflexivo. Além do mais, as Diretrizes determinam que as Instituições de Ensino Superior (IES) que fornecem tais cursos deverão contemplar, em seus Projetos Políticos Pedagógicos (PPPs), conteúdos curriculares presentes, nas disciplinas básicas e profissionalizantes, assim como desenvolver competências e habilidades gerais e específicas, dentre outros.

Ademais, isso não é diferente quando se trata da inserção de novos conhecimentos dentre as dinâmicas curriculares e suas ementas, em especial, a conteúdos relacionados à temática da violência nas IES, que aparecem de forma transversal nas disciplinas de Saúde da Criança, Saúde da Mulher e Saúde Pública ${ }^{(4)}$. Nesse sentido, demonstram ter déficits nos PPPs sobre 0 conteúdo de violência abordado no ensino superior, ressaltando que os profissionais não recebem formação adequada, quando se trata desse tema, o que se reflete na falta de habilidades exclusivas desses profissionais para uma assistência qualificada no que se refere ao atendimento a episódios de violência.

No que diz respeito a esse assunto, as IES são locais que permitem discutir e desenvolver estudos com essa perspectiva de mudanças, no perfil de formação profissional, o qual traz a enfermagem forense como importante papel ao combate a casos de violência ${ }^{(4)}$. Tal ciência foi consolidada em meados dos anos 1990, nos Estados Unidos da América (EUA) ${ }^{(5)}$, quando presta atendimento em distintos cenários em que a violência está presente, sejam vítimas, perpetradores e familiares envolvidos nessa perspectiva criminal ${ }^{(6)}$.
No Brasil, o Conselho Federal de Enfermagem (COFEN) estabelece a atuação do enfermeiro forense, por meio da Resolução $556 / 2017^{(7)}$, nos serviços carcerários, nas instituições psiquiátricas, dentre outros cenários em que a violência se encontra, destacando suas competências gerais e específicas. É importante salientar que a especialidade de enfermagem forense foi reconhecida, legalmente, pelos sistemas COFEN/Conselhos Regionais de Enfermagem pela Resolução 581/2018, que atualiza a lista de títulos de pós-graduação concedidos aos profissionais enfermeiros ${ }^{(8)}$.

Desse modo, para que os profissionais possam desempenhar seu papel nesses campos de atuação, deverão ser portadores do título de especialista, mestre (a)/ ou doutor (a) com ênfase em enfermagem forense, emitidos por IES reconhecida pelo Ministério da Educação (MEC) ou, por meio de Conselhos/Sociedades, para exercer suas atividades como enfermeiro forense no país ${ }^{(7)}$, sendo importante enfatizar que já existem programas de pós-graduação em enfermagem forense no Brasil( ${ }^{(4)}$.

Por outro lado, quando a prática de enfermagem forense é abordada nos cursos de graduação proporciona aos alunos um benefício do que se refere ao campo teórico-prático sobre esse assunto. Apesar disso, há certa necessidade de que esses conteúdos sejam incluídos nos currículos de enfermagem ${ }^{(9)}$. Além do mais, a falta de uma disciplina específica com enfoque em preparar os alunos a casos forenses faz com que as IES estabeleçam o interesse de introduzir essa temática no ensino de graduação, para despertar o interesse dos ingressantes, no campo da enfermagem forense, o que evidencia a falta de conhecimentos e de treinamentos nos cursos de Ensino Superior ${ }^{(10)}$.

Quando as IES fornecem tal disciplina/ou curso, é evidente que os alunos são mais informados, preparados e treinados para o enfrentamento em relação a episódios forenses no campo profissional, o que impacta, positivamente, em sua formação. Salienta-se a importância de se obter conhecimentos a respeito da enfermagem forense no ensino, bem como a inserção dos saberes forenses no currículo de enfermagem, além do fornecimento de cursos com certificação aos discentes, com o intuito de capacitá-los ainda na universidade ${ }^{(11)}$.

Nas IES públicas e privadas, os conteúdos de violência são oferecidos em disciplinas obrigatórias e optativas, no entanto aparecem de 
forma breve nas disciplinas por ciclo vital, como, por exemplo, nas disciplinas de saúde da criança e saúde da mulher, consequentemente, trazendo deficiência para a formação dos futuros profissionais de enfermagem ${ }^{(4)}$.

Acerca do impacto da violência na sociedade e no atendimento aos sobreviventes, agressores e familiares pelos profissionais de enfermagem nos diferentes cenários da prática clínica ${ }^{(6)}$, destaca-se a necessidade da existência de um profissional especializado para prestar cuidados e auxiliar o sistema legal, envolvendo crimes de natureza violenta ${ }^{(4)}$.

Portanto, é necessário que essa ciência seja abordada nas IES, com o propósito de desenvolver pesquisas a respeito desses conteúdos não só nas disciplinas oferecidas em sua dinâmica curricular, mas em explorar estudos para que os alunos estejam inseridos para uma melhor assistência às vítimas e aos agressores. Além do mais, irá contribuir para que os futuros enfermeiros se encontrem familiarizados com esse tema, para que seu atendimento seja de forma qualificada e humanizada, e que sejam um auxílio entre a saúde, o sistema social e o jurídico. Desse modo, esse estudo tem por objetivo identificar os conteúdos curriculares de enfermagem forense nos cursos de graduação em enfermagem.

\section{MÉTODO}

Trata-se de um estudo quantitativo, descritivo e exploratório, o qual foi realizado com coordenadores dos cursos de Enfermagem de 88 Instituições de Ensino Superior (IES) públicas e privadas, em que essas IES foram encontradas pelo Sistema Interativo do Ministério da Educação (e-MEC) que oferece, de forma gratuita, a consulta das IES em toda a região do país.

Quanto aos critérios de inclusão, foram aqueles coordenadores que aceitaram e respeitaram o período estipulado pelos pesquisadores. Já os de exclusão, foram cursos que não tinham iniciado turma, os que foram extintos, desativados, os que se recusaram a participar do estudo, além dos que não demonstraram interesse.

Entre os meses de setembro de 2018 e março de 2019, foi criado um questionário dicotômico e de perguntas abertas pelos pesquisadores, e validado empiricamente por quatro peritos na área de enfermagem forense. Os mesmos que validaram o instrumento, analisaram os itens propostos e, após esse refinamento, o questionário foi adaptado à ferramenta eletrônica do Google Forms, sendo de domínio público e gratuita.

O período de coleta de dados foi entre abril e agosto de 2019, sendo utilizado para análise dos dados, o Software Package for the Social Science (SPSS), para a estatística descritiva e inferencial, e o software R, para o Teste Exato de Fisher (5\% de significância), nas variáveis independentes (respostas positivas e negativas), e dependentes (IES públicas e privadas).

Para tal, a pesquisa foi aprovada pelo Comitê de Ética em Pesquisa da Universidade Federal de Alfenas, sob o Parecer: 2.893.206, CAAE: 97046718.0.0000.5142. Assim, os coordenadores receberam, por meio do instrumento elaborado pelo Google Forms, os Termos de Consentimento Livre e Esclarecido (TCLE) via eletrônica, e os juízes que validaram o instrumento também receberam uma cópia do TCLE, sendo reservado seu anonimato e sigilo entre os que compuseram este estudo.

\section{RESULTADOS E DISCUSSÃO}

De acordo com os resultados desta pesquisa, os conteúdos de enfermagem forense são ofertados, em sua maioria, nas IES privadas, situadas na região Sudeste. É importante realçar que, por meio da aplicação do Teste Exato Fisher, foi possível identificar quais conteúdos foram significantes, por meio dos cruzamentos entre as variáveis dependentes (conteúdos de enfermagem forense; IES públicas e privadas).

Assim, foi possível verificar a categoria sobre conteúdos curriculares de enfermagem forense, nas Instituições de Ensino Superior, para compor a discussão do presente estudo, os quais se referem à violência no trabalho, vítimas de desastre em massa, vítimas de ferimento por arma de fogo e branca, uso e abuso de álcool e outras drogas e pessoas em internações compulsórias.

\section{Conteúdos curriculares de enfermagem forense nas Instituições de Ensino Superior}

Ao cruzar as variáveis dependentes, utilizando o Teste Exato Fisher, os conteúdos de enfermagem forense que apareceram nas IES públicas e privadas, observou-se que foi possível identificar, por meio da Tabela 1, quais se destacaram em relação a sua significância $(p:<0,05)$, sendo que as variáveis selecionadas foram estatisticamente significantes e as IES privadas tiveram maiores destaques em relação às IES públicas. 
Quanto a esses conteúdos, a escolha foi dada por aqueles cujo valor estatístico foi significante, sendo relacionados à Avaliação/Intervenção de enfermagem a pessoas que sofreram violência no trabalho; Políticas e ações de prevenção/promoção de enfermagem a pessoas que sofreram violência no trabalho; Políticas e ações de prevenção/promoção de enfermagem a pessoas vítimas de desastre em massa; Avaliação/Intervenção de enfermagem a pessoas que sofreram ferimento por arma de fogo e branca; Avaliação/ Intervenção de pessoas sob uso e abuso de álcool e outras drogas; Avaliação/Intervenção de enfermagem em internações compulsórias; Políticas e ações de prevenção/promoção de enfermagem a pessoas em internações compulsórias.

Houve maior relevância nas IES privadas do que nas públicas, relacionados os conteúdos sobre vítimas de ferimento por arma de fogo e branca e pessoas sob uso e abuso de álcool e outras drogas, sendo que, nas IES privadas, esses conteúdos alcançaram um porcentual acima de $60 \%$. Já, nas públicas, atingiram uma média de $40 \%$.
Diante disso, no Brasil, o Ensino Superior de Enfermagem segue a DCN/ENF de 2001, que traz exigências de como os cursos de bacharelado em enfermagem devem ser regidos ${ }^{(3)}$. Nos últimos vinte anos (1995-2015) ${ }^{(12)}$, houve uma elevação muito significativa da oferta de cursos de Enfermagem, no Brasil, passando de 108 cursos para 887 (721,3\%), sendo que esse crescimento acelerado se deu por conta das IES privadas, tendo maiores concentrações nas regiões Sudeste (com maiores ofertas de cursos e vagas); Nordeste (fornece a metade deles); Sul, Centro-Oeste e Norte, que ofereceram em torno de 1.488 .445 vagas, e o crescimento dos que concluíram o curso, de 4.733 para 34.640 (632,0\%), totalizando $80,7 \%$ de enfermeiros já formados nesse período. Portanto, o estudo anterior corrobora os resultados da tabela 1 , pois, das IES que disseram oferecer tais conteúdos em suas respectivas instituições de ensino, as maiores concentrações de respostas foram nas IES privadas, enfatizando que, no país, o maior número dos cursos de enfermagem estão distribuídos nas instituições particulares do que nas estaduais e federais.

Tabela 1 - Distribuição da frequência dos conteúdos de enfermagem forense em relação às Instituições de Ensino Superior. Alfenas, MG, Brasil, 2019. ( $n=88)$.

\begin{tabular}{|c|c|c|c|c|}
\hline \multirow{2}{*}{ Conteúdos de Enfermagem Forense } & \multirow[b]{2}{*}{$\mathbf{N}$} & \multicolumn{3}{|c|}{ IES (\%)* } \\
\hline & & Privada & Pública & Valor-pt \\
\hline $\begin{array}{l}\text { 1. Avaliação/Intervenção de enfermagem a pessoas que sofreram violência no } \\
\text { trabalho }\end{array}$ & 38 & 50,0 & 20,0 & 0,021 \\
\hline $\begin{array}{l}\text { 2. Políticas e ações de prevenção/promoção pessoas que sofreram violência no } \\
\text { trabalho }\end{array}$ & 36 & 47,1 & 20,0 & 0,039 \\
\hline $\begin{array}{l}\text { 3. Políticas e ações de prevenção/promoção pessoas vítimas de desastre em } \\
\text { massa }\end{array}$ & 21 & 29,4 & 5,0 & 0,034 \\
\hline $\begin{array}{l}\text { 4. Avaliação/ Intervenção de enfermagem a pessoas vítimas de ferimento por } \\
\text { arma de fogo e branca }\end{array}$ & 53 & 66,2 & 40,0 & 0,042 \\
\hline $\begin{array}{l}\text { 5. Avaliação/Intervenção de enfermagem a pessoas sob uso e abuso de álcool e } \\
\text { outras drogas }\end{array}$ & 62 & 76,5 & 50,0 & 0,029 \\
\hline 6. Avaliação/Intervenção de enfermagem a pessoas em internações compulsórias & 22 & 30,9 & 5,0 & 0,019 \\
\hline $\begin{array}{l}\text { 7. Políticas e ações de prevenção/promoção a pessoas em internações } \\
\text { compulsórias }\end{array}$ & 22 & 30,9 & 5,0 & 0,019 \\
\hline
\end{tabular}

Fonte: Dados elaborados pelos autores. *IES: Instituições de Ensino Superior. †:Aplicação do Exato de Fisher. 
Em relação aos conteúdos de enfermagem forense sobre as vítimas de violência no trabalho, os profissionais de enfermagem sofrem graves consequências físicas e psíquicas relacionadas à violência no ambiente de trabalho, o que thes causa sérios problemas de saúde e de sofrimento mental ${ }^{(13)}$. Segundo o Conselho Regional de Enfermagem de São Paulo ${ }^{(14)}$, os tipos de violência que mais acometem esses profissionais foram agressões verbais e psicológicas, em que os pacientes é que perpetravam esse tipo de violência, em decorrência de falhas no atendimento, dentre outras situações. Portanto, os setores gerenciais, administrativos e acadêmicos, devem traçar propostas de prevenção/promoção da saúde e da segurança desses profissionais no seu campo profissional|(13).

No que se refere às Políticas e ações de prevenção/promoção de enfermagem a pessoas que sofreram violência no trabalho, compreende$\mathrm{se}^{(15)}$ que estão relacionadas a ações intervencionistas, a fim de prevenir esses episódios, em que a existência de programas voltados nessa perspectiva acabam ajudando os profissionais nos casos de sofrerem violência, em especial nos setores de urgência/emergência e psiquiátrico, bem como o desenvolvimento de novas políticas institucionais, que, além de combater a violência, possam trazer conforto e segurança para os profissionais de enfermagem no trabalho.

Quanto às Políticas e ações de prevenção/promoção pessoas vítimas de desastre em massa, existem, no Brasil, políticas públicas que visam à garantia dos direitos a essas vítimas, muitas vezes, podendo ser sobreviventes ou não, tendo o intuito de proteger sua dignidade e seus direitos, para minimizar seu sofrimento, dentre outros $^{(16-17)}$. Por outro lado, uma das estratégias de prevenção a ocorrências desses desastres é um planejamento assertivo da gestão nos serviços de saúde pública, principalmente das emergências, para evitar possíveis eventos catastróficos futuros, como, por exemplo, nos eventos esportivos, culturais, e assim por diante ${ }^{(18)}$. Os autores ${ }^{(18)}$ ainda enaltecem a importância do auxílio de outras esferas, como as da segurança, da logística e da saúde, entre outros; para que os profissionais, nesses setores, principalmente os da saúde, estejam preparados para agirem de forma capacitada e habilitada no que diz respeito a esses eventos em massa.
Os profissionais de enfermagem têm papel essencial na prestação de cuidados às vítimas de desastres em massa, junto à equipe multidisciplinar, para o qual a educação mostra ser uma ferramenta eficaz para capacitar e treinar na assistência direta a múltiplas vítimas ${ }^{(19)}$. Os Incidentes por Múltiplas Vítimas ${ }^{(20)}$, são um problema para o Sistema Único de Saúde (SUS), em especial, para a formação profissional do enfermeiro, sendo necessário explorar no ambiente acadêmico sua atuação frente a esses casos, principalmente no desenvolvimento de habilidades e competências profissionais. Assim, é fundamental que o professor seja um mediador para colaborar nesse processo de ensinoaprendizagem relacionado a esses cenários em massa.

No que diz respeito à Avaliação/Intervenção de enfermagem a pessoas vítimas de ferimento por arma de fogo e branca ${ }^{(21)}$, sua maior prevalência foi de vítimas jovens do sexo masculino, que estavam sóbrias, em que o assalto teve maiores registros, seguido de homicídios ocasionados nos finais de semana. Os procedimentos clínicos realizados às vítimas de traumas foram desde curativos até oxigenoterapia, sendo utilizados protocolos específicos para 0 atendimento aos traumatizados, em que esses tipos de violência geram gastos não só para o setor de saúde, como para o econômico e o social.

Além disso, esses autores publicaram outro estudo no mesmo ano sobre o perfil epidemiológico das vítimas por arma branca ${ }^{(22)}$, segundo o qual esse perfil era de jovens do sexo masculino, no entanto a maior incidência dessas ocorrências foi por agressões interpessoais e tentativa de suicídio, em que essas vítimas estavam sóbrias no ato violento, e os procedimentos clínicos foram os mesmos citados no estudo anterior ${ }^{(21)}$. Os autores ${ }^{(22)}$ ressaltam que o local desses ferimentos ocorreu nas regiões do abdome, do tórax e dos membros inferiores, salientando a necessidade de combater esse tipo de violência, por meio de ações de promoção à saúde e educação nesses cenários de vítimas de traumas.

Quanto ao campo da saúde mental sobre Avaliação/Intervenção de enfermagem a pessoas sob uso e abuso de álcool e outras drogas ${ }^{(23)}$, os enfermeiros que atuam na Saúde da Família perceberam que a temática sobre esse assunto é um problema de saúde pública e social, e que os 
Agentes Comunitários de Saúde e os familiares são pontes para detectar esses indivíduos. Entretanto, os autores ${ }^{(23)}$ destacam que há uma certa dificuldade desses profissionais em intervir no que diz respeito ao tratamento na própria unidade de saúde, bem como aos usuários sobre seu problema relacionado ao consumo, em que os enfermeiros disseram que a vontade e escolha tem que partir do próprio indivíduo, sendo que há uma certa resistência à adesão ao tratamento, da não capacitação desses profissionais, e da oferta de grupos terapêuticos para o enfretamento desses casos.

Em relação aos conteúdos de enfermagem forense referentes à Avaliação/intervenção de enfermagem e Políticas e ações de prevenção/promoção a pessoas em internações compulsórias, as enfermeiras da saúde mental compreendem o atendimento a essas internações (compulsórias e involuntárias), sendo necessária uma avaliação do caso, para que as necessidades individuais possam ser atendidas, em especial no contexto familiar e sociocultural, porém, muitas vezes, os leitos estão superlotados. Isso faz com que esses familiares busquem auxílio do próprio setor judiciário para intervir nessa situação, causando sofrimento entre os pacientes e os familiares ${ }^{(24)}$.

Além do mais, os autores ${ }^{(24)}$ frisam que as internações deveriam ser pela própria vontade do usuário, mas isso nunca acontece, o que gera consequências para a saúde, como angústia e ansiedade. Os profissionais, quando o usuário chega a essas circunstâncias, sentem certa frustração, por acharem que não conseguiram dar integralmente uma assistência de qualidade e, por consequência, gerando uma má comunicação entre a equipe que assiste esses pacientes.

Uma das alternativas que poderiam resolver esses conflitos nos serviços de saúde mental e psiquiátrico seria utilizar da Educação Permanente como uma ferramenta para gerar essas discussões no ambiente de trabalho, como, por exemplo, ouvir o que esses profissionais têm a dizer sobre suas indagações e preocupações, para tentar manter bom relacionamento interpessoal entre a equipe $^{(24)}$.

No que diz respeito à Lei n. 10.216 de 6 de abril de $2001^{(25)}$, que dispõe sobre a proteção e os direitos das pessoas portadoras de transtornos mentais, a internação compulsória é vista como determinada pela justiça, sendo que o Estado é responsável por desenvolver políticas de ações de promoção e de prevenção no atendimento a essa população, bem como a devida participação da sociedade e da família, entre outros direitos.

Portanto, é notória a participação da enfermagem nesses cenários da violência como foi vista nessa categoria, e de como esses conteúdos são abordados nas IES privadas. É importante enfatizar que é preciso que esses conteúdos sejam abordados nas escolas/cursos de enfermagem no país, como também de se trabalhar com a enfermagem forense ainda na graduação. Além disso, os próprios coordenadores e docentes deverão rever como esses conteúdos relacionados à enfermagem forense estão sendo inseridos no projeto pedagógico do curso, pois, assim, se fará que o aluno consiga assimilar com o que está aprendendo em sala de aula, para que seja aplicado na prática do estágio curricular.

\section{CONCLUSÃO}

Foi possível identificar que os conteúdos curriculares de enfermagem forense nos cursos de graduação em enfermagem são mais oferecidos nas IES privadas, do que nas públicas. Para tal, mostra-se fundamental que as escolas de enfermagem incluam, em sua dinâmica curricular, conteúdos específicos de enfermagem forense, tanto nas disciplinas que aparecem de forma transversal, como nas de saúde da criança e do adolescente, na saúde da mulher, dentre outras disciplinas. Nesse sentido, os professores conseguirão trabalhar sobre essa temática com os alunos, articulando a teoria com a prática, como havia evidenciado na literatura a relevância da existência de uma disciplina sobre a enfermagem forense no ensino superior de enfermagem.

Portanto, sugerem-se novos estudos sobre a enfermagem forense na saúde da criança, saúde da mulher, saúde do adulto, saúde do idoso, saúde coletiva, pronto atendimento e na saúde mental em que, possivelmente, esses conteúdos também possam aparecer, para que, assim, seja viabilizada de forma mais articulada a assistência de enfermagem a esse cenário ainda no processo de formação do futuro enfermeiro, de modo a despertar o interesse do aluno de entrar no campo das ciências forenses como, por exemplo, no desejo de querer fazer uma especialização em enfermagem forense.

Embora os conteúdos tenham aparecido de forma vaga pelo cruzamento dos dados, houve poucas IES que aceitaram participar do estudo, sendo preciso prolongar o período da coleta de dados, pois o coordenador do curso possui várias 
atividades de trabalho, para que este consiga dar sua contribuição da melhor forma possível. Desse modo, os estudos demonstraram que são poucas as pesquisas, no Brasil, sobre a área do ensino na enfermagem forense, $o$ que sugere $o$ desenvolvimento de novos estudos nessa mesma perspectiva.

\section{REFERÊNCIAS}

1- Bezerril MS, Chiavone FBT, Lima JVH, Vitor AF, Ferreira JM, Santos VEP. Ensino de enfermagem: uma análise do conceito segundo o método evolucionário de Rodgers. Esc Anna Nery 2018;22(4):1-6. DOI: 10.1590/2177-9465-ean2018-0076

2- Salvador PTCO, Alves KYA, Rodrigues CCFM, Costa TD, Assis YMS, Santos VEP. Ensino de enfermagem: caminho das pesquisas. Rev Enferm UERJ 2017;25:1-7. DOI: 10.12957/reuerj.2017.21469

3- Brasil. Resolução CNE/CES no 3, de 7 de novembro de 2001. Institui Diretrizes Curriculares Nacionais do Curso de Graduação em Enfermagem. Diário Oficial da União 2001.

4- Martins DC, Gois OJO, Silva JOM, Rosa MPRS, Gonçalves MC. Violência: Abordagem, atuação e educação em enfermagem. Ciências Biológicas Saúde UNIT 2017 [citado em 7 out 2019]; 4(2):155$68 . \quad$ Available in: https://periodicos.set.edu.br/index.php/caderno biologicas/article/viewFile/4603/2501

5- Drake SA, Koetting C, Thimsen K, Downing N, Porta $\mathrm{C}$, Hardy $\mathrm{P}$, et al. Forensic nursing state of the science: Research and practice opportunities. J Forensic Nurs. 20181;14(1):3-10. DOI: 10.1097/JFN.0000000000000181

6- Lynch VA. Forensic nursing science: Global strategies in health and justice. Egypt J Forensic Sci. 2011;1(2):69-76. DOI: 10.1016/j.ejfs.2011.04.001

7- Brasil. Resolução no 581, de junho de 2018. Procedimentos de título de pós-graduação lato e stricto sensu concedido a enfermeiros e lista as Especialidades. Diário Oficial Brasília 2018;137(1):119.

8- Brasil. Resolução no 556, de 23 de agosto de 2017. Regulamenta a atividade do enfermeiro forense no Brasil. Brasília: COFEN; 2017.

9- Cunha $M$, Libório $R$, Coelho $M$. Knowledge questionnaire over forensics nursing practices. Procedia Soc Behav Sci. 2016;217:1089-97. DOI: 10.1016/j.sbspro.2016.02.118

10- Topçu ET, Kazan EE. The opinions of senior nursing students about forensic nursing. Egypt J
Forensic Sci. 2018;8(16). DOI: 10.1186/s41935018-0045-y

11- Ozden D, Ozveren H, Yılmaz i. The impact of forensic nursing course on students' knowledge level on forensic evidence. J Forensic Leg Med. 2019;66(6):86-90.

DOI: 10.1016/j.jflm.2019.06.012

12- Vieira ALS, Moyses NMN. Trajetória da graduação das catorze profissões de saúde no Brasil. Saúde Debate 2017;41(113):401-14. DOI: 10.1590/0103-1104201711305

13- Bordignon $M$, Monteiro MI. Violência no trabalho da enfermagem: Um olhar às consequências. Rev Bras Enferm. 2016;69(5):9969. DOI: 10.1590/0034-7167-2015-0133

14- Conselho Regional de Enfermagem de São Paulo. Violência no trabalho: Guia de prevenção para os profissionais de Enfermagem. 2017 [citado em 7 out 2019]; 1-42 Available in: https://portal.coren-sp.gov.br/wp-

content/uploads/2018/01/PDF-site-2.pdf

15- Pereira CAR, Borgato MH, Colichi RMB, Bocchi $\mathrm{SCM}$. Institutional strategies to prevent violence in nursing work: An integrative review. Rev Bras Enferm. 2019;72(4):1052-60. DOI: 10.1590/00347167-2018-0687

16- Organização Pan-Americana da Saúde. Gestão de cadáveres após desastres: Manual para equipes de primeira resposta no terreno. 2a ed. ver. Washington: OPAS; 2016.

17- Furtado JR, Silva MS (Orgs). Proteção aos direitos humanos das pessoas afetadas por desastres. Florianópolis: CEPED UFSC; 2014.

18- Castro CF, Simões DCM, Delamarque EV, Pepe VLE. Eventos de massa, desastres e Saúde Pública. Ciênc Saúde Coletiva 2014;19(9): 371730. DOI: 10.1590/1413-81232014199.02282014 19- Mello CM, Witt RR, Dorneles EL, Marin SM. A enfermagem no atendimento em desastres e em eventos múltiplas vítimas. Vittalle 2013 [citado em 7 out 2019]; 25(1):37-44. Available in: https://periodicos.furg.br/vittalle/article/viewFile /6018/3705

20- Salvador PTC O, Dantas RAN, Dantas DV, Torres G V. A formação acadêmica de enfermagem e os incidentes com múltiplas vítimas: Revisão integrativa. Rev Esc Enferm USP 2012;46(3):74251. DOI: $10.1590 /$ S0080-62342012000300029 21- Zandomenighi RC, Martins EAP, Mouro DL. Ferimento por projétil de arma de fogo: Um problema de saúde pública. Rev Min Enferm. 2011 [citado em 7 out 2019]; 15(3):412-20. Available in: http://www.reme.org.br/artigo/detalhes/53 
22- Zandomenighi RC, Mouro DL, Martins EAP. Ferimento por arma branca: Perfil epidemiológico dos atendimentos em um pronto socorro. Rev RENE 2011 [citado em 7 out 2019]; 12(4):669-77. Available in: http://www.revistarene.ufc.br/revista/index.php /revista/article/download/281/pdf

23- Souza LM, Pinto MG. Atuação do enfermeiro a usuários de álcool e de outras drogas na Saúde da Família. Rev Eletrônica Enferm. 2012;14(2):374-83. DOI: 10.5216/ree.v14i2.11245 24- Xavier MS, Terra MG, Schimith MD, Leite MT, Kruse MHL, Arnemann CT. The understanding of nurses who work in mental health regarding compulsory and involuntary hospitalization. Esc Anna Nery 2017;21(3):18. DOI: 10.1590/21779465-ean-2016-0262

25- Brasil. Lei n. 10.216 de 6 de abril de 2001. Dispõe sobre a proteção e os direitos das pessoas portadoras de transtornos mentais e redireciona o modelo assistencial em saúde mental. Diário Oficial da União 2001;1:2.

Nota: Artigo extraído da dissertação - Enfermagem forense em cursos de graduação em enfermagem, apresentado ao Programa de Pós-Graduação em Enfermagem da Universidade Federal de Alfenas, em 2020. O presente trabalho foi realizado com o apoio da Coordenação de Aperfeiçoamento de Pessoal de Nível Superior (CAPES) Código de Financiamento 001.

Recebido em: 30/12/19

Aprovado em: 16/04/20

Endereço de correspondência:

Jhuliano Silva Ramos de Souza

Rua da Liberdade, 72 - Vila Betânia

CEP: 37137-090 - Alfenas/MG - Brasil

E-mail:jhulianoramoz@hotmail.com 